Full citation: Bacic, B., Kasabov, N.K., MacDonell, S.G., \& Pang, S. (2008) Evolving connectionist systems for adaptive sport coaching, in Proceedings of the Fourteenth International Conference on Neural Information Processing (ICONIP'07). Kitakyushu, Japan, Springer (Lecture Notes in Computer Science v.4985), pp.416-425.

doi: 10.1007/978-3-540-69162-4_43

\title{
Evolving Connectionist Systems for Adaptive Sport Coaching
}

\author{
Boris Bacic, Nikola Kasabov, Stephen MacDonell, and Shaoning Pang \\ KEDRI and SERL \\ School of Computing and Mathematical Sciences \\ Auckland University of Technology (AUT) \\ Private Bag 92006, Auckland 1142, New Zealand \\ \{bbacic,nkasabov, smacdone, spang\}@aut.ac.nz
}

\begin{abstract}
Contemporary computer assisted coaching software operates either on a particular sub-space of the wider problem or requires expert(s) to operate and provide explanations and recommendations. This paper introduces a novel motion data processing methodology oriented to the provision of future generation sports coaching software. The main focus of investigation is the development of techniques that facilitate processing automation, incremental learning from initially small data sets, and robustness of architecture with a degree of interpretation on individual sport performers' motion techniques. Findings from a case study using tennis motion data verify the prospect of building similar models and architectures for other sports or entertainment areas in which the aims are to improve human motion efficacy and to prevent injury. A central feature is the decoupling of the high-level analytical architecture from the lowlevel processing of motion data acquisition hardware, meaning that the system will continue to work with future motion acquisition devices.
\end{abstract}

Keywords: Classification, Coaching Rule, CREM, Coaching Scenario, ECOS, EFuNN, iB-fold, Feature Extraction, Local Personalised Global Knowledge Integration, Orchestration, Weighted Sum.

\section{INTRODUCTION: COMPUTER ASSISTED SPORT COACHING}

Contemporary computer assisted sport coaching software can be divided into two major categories:

1. Relatively expensive software and hardware solutions designed to be operated across domains by experts in the areas of Biomechanics or Physiotherapy [1, 2].

2. Specialised software for a particular sport's subdomain that is affordable to the sport's enthusiasts and that does not need to be operated by a professional domain expert (although some initial and follow-up expert assistance may be recommended). In general, such solutions are intended $^{1}$ to provide feedback typically as an animated/video replay or as a report containing measured values compared with 'optimal' biomechanical key values [3, 4]. In the case of entertainment applications (i.e. videogames and sport simulations) a player can interact with a virtual environment [5] using a motion sensitive input device such as the Wii controller (http://www.nintendo. com/overviewwii). Intended coaching may occur through repetitive interaction with the environment.

At present, commercial software development in this domain is restricted by the cost of $3 \mathrm{D}$ motion data acquisition relative to precision, accuracy, noise, sampling frequency and robustness to occasional data loss. Other restrictive and undesired factors that contribute to limited progress are: the degree of obtrusiveness, environmental and operational restrictions (e.g. autonomy of unsupervised operation, robustness to shock, vibration and ambient operating ranges), the often lengthy time required to set up the environment with initial settings, and the resulting extent of expert involvement. Examples of computational and operational limitations impeding the development, adoption and/or success of computer assisted coaching tools have also been discussed in [6].

In general, these limitations can be considered in terms of two major groupings:

1. The first category of drawbacks (of present sport coaching software) consists of excessive user operation/intervention, required sport domain knowledge, and the advanced nature of the computer equipment (e.g. motion data transfer, setting up recording environment, digitising and "slicing"

\footnotetext{
${ }^{1}$ i.e. limited aspects of non-evolvable coaching compared to human coaching. It is also not intended to replace, but to complement human supervised coaching aspects.
} 
video, annotations and measurements and measurements interpretation).

2. The second category of drawbacks relates to limitations in motion data processing e.g. lack of adaptability, inability to learn from data, and insufficient or limited results interpretations and personalisation.

A level of informed speculation (i.e. by extending Moore's Law) enables us to predict that near-future ICT technology for obtaining real-time, high precision motion data will be more ubiquitous and more affordable. The same level of speculation applied to Human Computer Interaction (HCI) devices suggests that it will soon be possible to provide multimedia-rich feedback to learners from systems that are capable of assessing human motion. Such predictions provide the necessary infrastructural support to underpin the feasibility of generic evolving computational models for assessing human motion as a separate layer from low-level motion data processing and HCI management layers.

\section{PROPOSED ADAPTIVE COACHING SYSTEM}

The initial stages of this study have been focused on scoping an appropriate experimental system design and choosing an initial case study in tennis.

\subsection{Motivation for Adaptive Sports Coaching System}

Bridging the interdisciplinary gap between Sport Biomechanics and the application area of Evolving Connectionist Systems (ECOS) [7], a novel coaching system based on a robust and adaptive software architecture should have the following capabilities:

- Adaptation to new playing motion data (e.g. swing techniques) that can be incrementally presented,

- Rule based knowledge interpretation from motion data, including knowledge insertion and extraction,

- Personalised (swing) technique assessment modelling and personalised coaching where new modules and connections can be introduced at any stage of the system's operation including adaptation to new (swing) rules and variables,

- Knowledge separation into Personalised, Global and Environmental levels (e.g. Coaching Scenarios (CS) and individual coaching approaches),

- $\quad$ Ability to work with initially small data sets, and with incomplete Global and Environmental knowledge,

- $\quad$ Supervised, Unsupervised, Incremental and Online learning.

\subsection{Evolving Coaching: An Adaptive Tennis Coaching System}

In 'traditional' (i.e. human assisted) sport coaching, a coach can correct and potentially improve an observed learner's motion technique by communicating a set of relevant coaching rules combined with intervention instruction(s) to the learner. In the system proposed here, adherence or otherwise to each relevant coaching rule is observed and assessed individually in a Coaching Rule Evaluation Module (CREM), as shown in Fig. 1.

Section 2.4 further explains the Orchestration paradigm as a set of individual CREMs collectively assessing human motion.

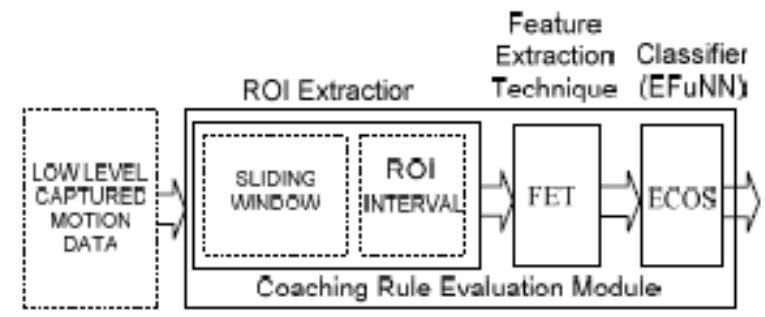

Fig. 1. CREM block diagram and stages of data processing. CREMs are responsible for classification of both temporal and spatial qualitative observations by a coach, although represented/expressed numerically. A process of transforming a temporal/spatial Region of Interest (ROI) to quantitative observations needed for machine learning classification is referred to here as Feature Extraction Technique (FET).

CREM motion data processing occurs in multiple stages:

1. The first stage of CREM processing involves the automated extraction of the Region of Interest (ROI). ROI automation in a stand-alone CREM (Fig. 1) would include the recognition of tennis shots and their ROI extraction from time series of 3D tennis data (section 3.1). A two-staged algorithm structure [8] allows hyper threading implementation for quick detection and ROI extraction.

2. In the Feature Extraction Technique (FET) stage, the system is responsible for mathematically transforming the ROI into a set of the most discriminative key values representing each shot. Compared to human qualitative assessment of a shot, a coach would typically focus on constituent time sub-segments of the shot. Within each time subsegment, the coach would analyse observed temporal (e.g. hip rotation leading shoulder turn) or spatial (e.g. swing width) key features to assess adherence to a particular coaching rule (or heuristic).

3. In the final stage, an ECOS classifier module is responsible for numerically assessing/evaluating adherence to a particular observed coaching rule. As the output of the last stage of CREM internal processing, an ECOS module provides the overall CREM output as and if required, to supply feedback to the learner and to augment the system's coaching knowledge (Fig. 5). 


\subsection{Evolving Fuzzy Neural Network Architecture (EFuNN)}

ECOS has been developed to address several of the perceived disadvantages and limitations of traditional connectionist systems - by comparison, ECOS are resilient to over-training, they learn and adapt their structure quickly, and they are far more resistant to catastrophic forgetting [7]. Paradoxically, it is these very advantages that cause some of ECOS' disadvantages. Since they deal with new examples by adding nodes to their structure, they rapidly increase in size and can become unwieldy if no aggregation or pruning operations are applied. They also have some sensitivity to their parameters, which require constant adjustment for optimum performance.

An ECOS network always has at least one evolving layer, such as the middle layer shown in the Evolving Fuzzy Neural Network depicted in Fig. 2. This is the layer that will grow and adapt itself to the incoming data, and is the layer with which the learning algorithm is most concerned.

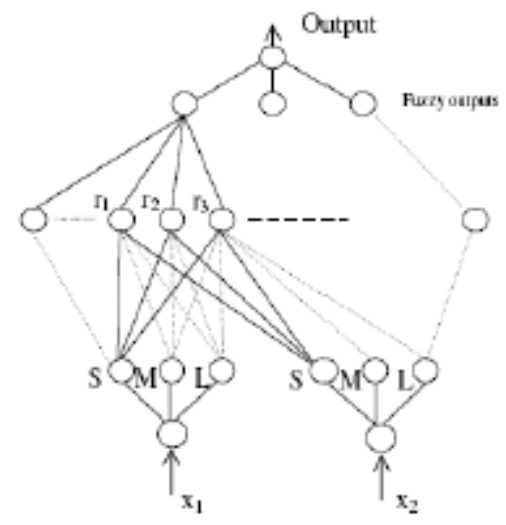

Fig. 2. EFuNN structure as an ECOS classifier with two inputs and one output ([9], p.677)
Although there are a growing number of ECOS implementations $[7,9,10]$, that chosen here is a relatively simple solution. It would be an informative future exercise to assess which ECOS model works better for a particular CREM module. However, several have highvolume data requirements (in order to perform parameter optimisation) which could limit their viability in lowvolume data applications.

A simple implementation of ECOS was achieved using EFuNN, Fig. 2, with its ability to work with initially small data sets and to extract knowledge as a set of fuzzy rules. Selecting the Membership Functions (MF) and their number in a given EFuNN would depend on the particular CREM task (e.g. if an expert would say "a player's wrist can be too far away from the body but never too close, when hitting ...” then two MFs would be adequate for a CREM assessing swing width).

\subsection{CREM Orchestration}

To accommodate diversity in Coaching Scenarios (CS) and in human coaches' qualitative analyses, a method for their orchestration has been proposed, as depicted in Fig. 3, Proposed in [12], a weighted sum ECOS Architecture would support the implementation of Global, Local (i.e. Environmental - as CS and different coaching approaches) and Personalised modelling introduced in [9]. Each CREM uses its own features sub-set. Skill and Weights parameters (from Fig. 3) can also be stored in any of the Personalised, Environmental/CS or Global databases.

Automated shot extraction for each CREM is performed before the Rule Module Selector to avoid computational overlapping. Each selected CREM participating in the shot evaluation automatically extracts only the temporal sub set (sub event) needed for its own feature extraction.

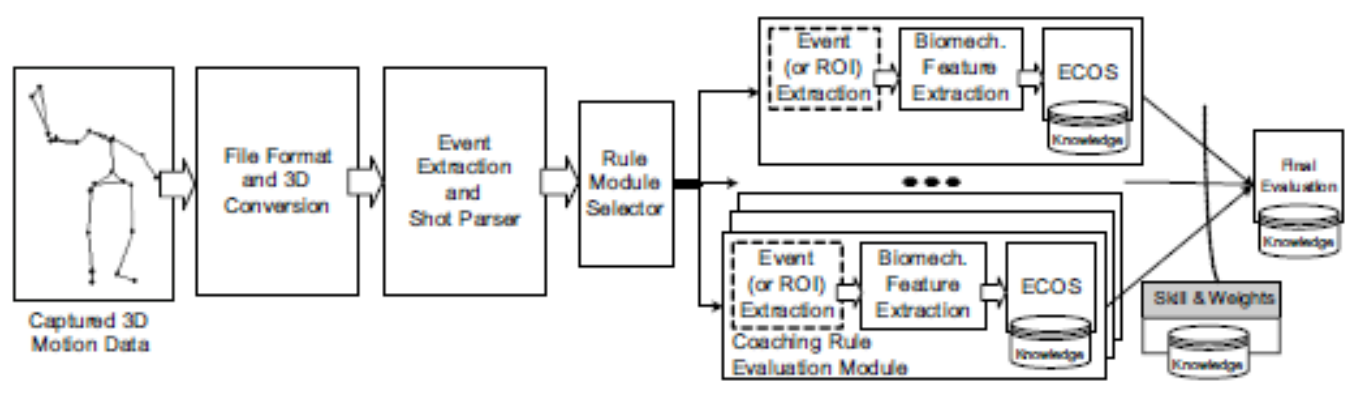

Fig. 3. CREM Orchestration and modular stages of data processing

\section{EXPERIMENTATION AND SIMULATION}

Aspects of experimental design are now addressed, followed by a description of the outcomes of different activities that led from ideas to specific results. Tennis data were recorded whereby an expert coach mimicked styles representative of beginner players. To ensure that data samples were sufficiently represented in typical swing style clusters, the expert's mimicking was verified by two independent coaching experts in two stages i.e. during the recording stage and later on, in a subsequent "blind review" manner examining only captured motion data in the from of an animated "stick model” (Fig. 4).

\subsection{Motion Data Set Acquisition}

The human motion dataset was recorded using 3D marker positions in a time series at a sampling frequency of $50 \mathrm{HZ}$ (or fps) and one millimetre resolution. To capture $3 \mathrm{D}$ motion using multi-camera infra-red (IR) recording 
technology in IR spectrum, a set of retro-reflective markers was attached to selected anatomical landmarks of the player's body. By defining the markers' topology a "stick model" was created to represent a human body. Animated visualization of the stick figure (Fig. 4) approximating a human swinging a racquet - was sufficient for the expert coach to verify the mimicking process, to provide output labels for machine classification and to give qualitative analysis that influenced high level architecture design. The expert's involvement was also required in defining players' expected skill level relative to the operation of a set of CREM for a given Coaching Scenario (CS).

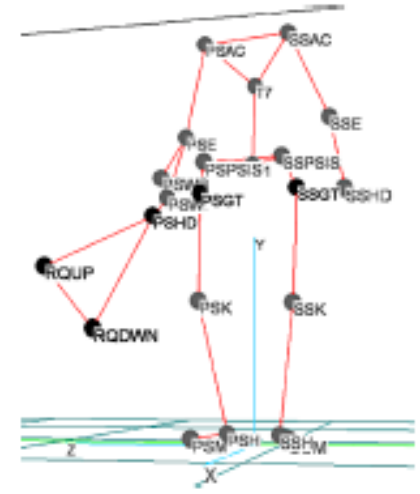

Fig. 4. A “stick figure” representing a tennis player holding a racquet

\subsection{Processing and Validation}

After qualitative analysis of critical key features on which a tennis coach would focus their attention, the first testable hypothesis was framed. This aimed to quantify the 'most critical' critical key features that could coarsely discriminate between tennis swings e.g. "good" or "bad" style for a forehand swing. A hypothesis asserting the correlation between motion of the racquet hitting surface (around the point of impact) and the player's body motion was chosen to be tested as the first and the strongest hypothesis for that purpose. The first prototype, constructed to enable the testing of the hypothesis (published in [11]), did not require adaptive learning. It was designed using a Radial basis function (RBF) neural network from the open source Netlab toolbox (http://www.ncrg.aston.ac.uk/netlab/down.php) and accompanying book [12].

The need for the experimental work to minimise generalisation error using a relatively small dataset (40 samples) in spite of potentially high dimensionality of the problem space demanded rigorous consideration in the following areas (see also Table 3):

1. Choosing the validation method

2. Exploring the benefits of expert cognitive preclustering

3. Evolving architecture design.

Training and classification evaluation of the first experimental prototype was undertaken using the "leaveone-out” cross-validation method (Table 1).
Table 1. Leave-one-out cross-validation. To ensure that over-fitting was avoided, a sub-optimal model utilising 2 hidden neurons was tested and is included in the results. Due to incomplete time series markers position data, the original set of 19 extracted forehand shots was further reduced to 14 .

\begin{tabular}{rrl}
\hline Number of Input Vectors & 14 \\
\hline Number of Cross-validations & 20 \\
Classification Accuracy & 2 hidden neurons & $66.4[\%]$ \\
& 3 hidden neurons & $99.9[\%]$ \\
& 4 hidden neurons & $99.9[\%]$ \\
\hline
\end{tabular}

During the design stages for subsequent CREMs, different validation methods were also considered and evaluated, taking into account the expert's familiarity with the data and probability for error. The stochastic relation between data and validation method error as incident prediction $P(C)$ has been investigated in [13]. The data set was pre-clustered into eight groups by the expert applying Gestalt observation model in biomechanics [1]. An erroneous validation incident example would occur where an entire cluster is allocated to the test portion of the dataset. The probabilistic formula (1) for single iteration data split incident calculation in hold-out validation method has been confirmed by comparing large number $\left(n \times 10^{6}\right)$ of simulation results.

$$
P(C)=\frac{\left(\begin{array}{c}
j \\
k
\end{array}\right)\left(\begin{array}{ll}
n & -j \\
m & -k
\end{array}\right)}{\left(\begin{array}{l}
n \\
m
\end{array}\right)}
$$

Where:

$P(C)$... Probability of event $C$; defined as $P(k$ cluster samples in test dataset)

$j$... size of observed cluster

$k$... number of samples in test data from observed cluster

$n$... size of the data sample

$m$... size of the test dataset portion

To avoid $P(C)$ incidents further expert cognitive preclustering cross-validation algorithms (e.g. iB-fold [14], as modified leave-v-out) have been used.

\subsection{Model Integration}

The next stage of the research was focused on the automated extraction of the Region of Interest (ROI), including recognition of each tennis shot and its extraction from time series 3D data [8]. Compared to human expert shot extraction, the average prediction for the first frame number in the automated shot extraction was 0.789 frames slower; and for end swing the difference was -0.16 frames - i.e. predicting the end of swing was $3.2 \mathrm{~ms}$ earlier. The duration of a frame $=0.02$ sec. Detailed results are given in Table 2.

By merging the outcome of both experimental studies into a single CREM prototype Fig. 1, the further CREMs were designed to operate as components of the integrated coaching system. 
Table 2. Experimental results on automated Forehand ROI extraction compared to expert's manual ROI extraction. Number of extracted Forehand shots $=19$.

\begin{tabular}{rccccc}
\hline & $\begin{array}{r}\text { Duration } \\
\text { [frames] }\end{array}$ & $\begin{array}{r}\text { Start frame } \\
\text { Delta }\end{array}$ & $\begin{array}{r}\text { End frame } \\
\text { Delta }\end{array}$ & $\begin{array}{r}\text { Duration } \\
\text { [frames] }\end{array}$ & $\begin{array}{l}\text { Delta } \\
\text { duration }\end{array}$ \\
\hline Average & 8.263 & 0.789 & -0.16 & 7.316 & 0.947 \\
Max & 13 & 3 & 1 & 12 & 4 \\
Min & 5 & 0 & -1 & 5 & -1 \\
Median & 8 & 1 & 0 & 6 & 1 \\
Range & 8 & 3 & 2 & 7 & 5 \\
\hline
\end{tabular}

\subsection{Rule Extraction}

The other CREMs were designed using ECOS, as per the overall architecture shown in Fig 1. By using EFuNN from Fig. 2, it was possible to extract knowledge as a set of fuzzy rules and apply further translation of that knowledge into a form closer to that provided by a human coach (rather than as a large number of rules that were potentially difficult to comprehend). To make effective use of these rules the coaching principle "less is more" was taken into account. The system was designed with the aim of reducing the rule set to key or high-priority issues - ideally in most CS a learner would address one improvement at a time.

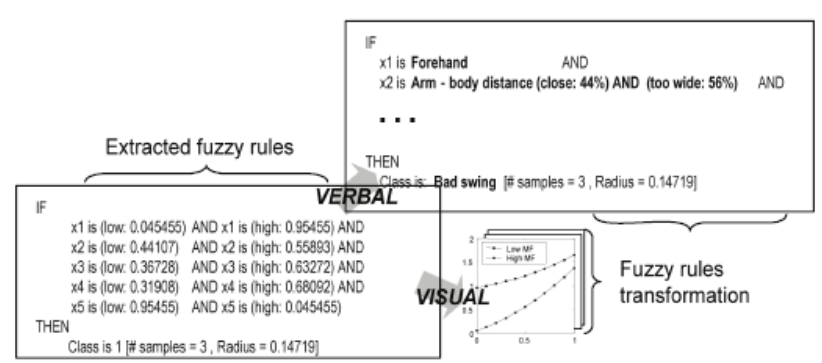

Fig. 5. CREM block diagram and stages of data processing. From human perspective a player can focus on individual improvement of particular coaching rule over a period of time.

\section{CONCLUSION AND FUTURE WORK}

The methodology utilised in this work is shown in summary form in Table 3. Apart from contributing to the application area of ECOS and bridging the discipline with research in biomechanics, the methodology has parallels in diverse areas such as medicine and bioinformatics e.g. Inductive vs Transductive approach, Global, Local and Personal modelling in Bioinformatics [9] with CREM Orchestration. Similar to the approach shown in Table 3, another activity-driven approach was independently developed in the UK as the Standard Integrative Systems Biology Approach [15].

Table 3. Summary of main activities that have led from ideas to specific results

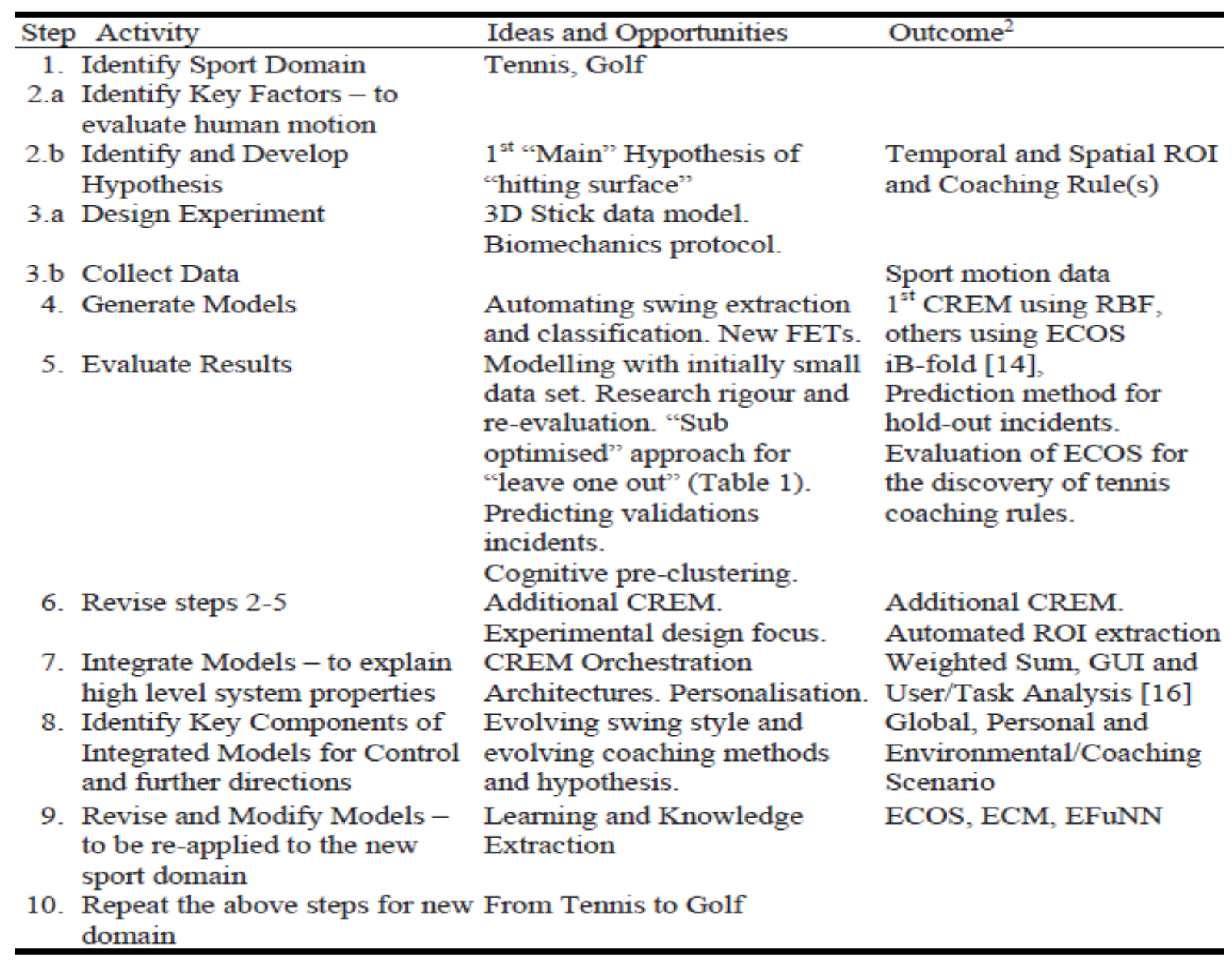


The modelling -follow up case study - of revisited models (step 9, Table 3) from tennis in the golf domain will soon be completed based on an already collected large data set of golf swings. Further advancement in presenting fuzzy rules to humans will promote applicative advancement in the area of neuro-fuzzy systems. In conclusion, for human motion applications, in spite of the constraints associated with state of the art technology of motion data acquisition (time consuming, labour intensive, expensive) the favourable experimental results to date give promise of an interesting and innovative future research area.

\section{ACKNOWLEDGMENTS}

As the first author, I wish to express my appreciation to list of people who also inspired and offered their support: Dr. Zeke S. Chang, Prof. Patria Hume, Prof. Takeshi Yamakawa, Gordon Grimsey and Petar Bačić. Also I wish to acknowledge contributors' extended support and creating opportunities beyond $\mathrm{PhD}$ supervision. Tennis data was collected from "Peharec" Polyclinic for physical therapy and rehabilitation, Pula, Croatia. Golf data was collected from AUT Golf Driving Range. AUTEC ethics data collection approval, number 06/105.

\section{REFERENCES}

1. Knudson, D.V., Morrison, C.S.: Qualitative Analysis of Human Movement. Human Kinetics, Champaign (2002)

2. SiliconCOACH PRO. SiliconCoach Ltd., Dunedin (2005)

3. SmartSwing. SmartSwing Inc., Austin (2005)

4. Leadbetter Interactive. Interactive Frontiers, New Hudson (2005)

5. Master Game List. Nintendo of America Inc., Redmond, WA, vol. 2007 (2007)

6. Bacic, B.: Bridging the Gap between Biomechanics and Artificial Intelligence. In: Schwameder, H., et al. (eds.) International Symposium on Biomechanics in Sports - ISBS 2006, Department of Sport Science and Kinesiology, vol. 1, pp. 371-374. University of Salzburg, Austria, Salzburg, Austria (2006)

7. Kasabov, N.K.: Evolving Connectionist Systems: Methods and Applications in Bioinformatics, Brain Study and Intelligent Machines. Springer, London (2002)

8. Bacic, B.: Towards a Neuro Fuzzy Tennis Coach: Automated Extraction of the Region of Interest (ROI). In: International Conference on Fuzzy Systems (FUZZ-IEEE) and International Joint Conference on Neural Networks (IJCNN), vol. 2, pp. 703-708. IEEE, Budapest, Hungary (2004)

9. Kasabov, N.: Global, Local and Personalised Modeling and Pattern Discovery in Bioinformatics: An Integrated Approach. Pattern Recognition Letters 28, 673-685 (2007)

10. Kasabov, N.: Adaptation and Interaction in Dynamical Systems: Modelling and Rule Discovery through Evolving Connectionist Systems. Applied Soft Computing 6, 307-322 (2006)

11. Bacic, B.: Automating Systems for Interpreting
Biomechanical 3D Data Using ANN: A Case Study on Tennis. In: Kasabov, N., Chan, Z.S.H. (eds.) 3rd Conference on Neuro-Computing and Evolving Intelligence - NCEI 2003. Knowledge Engineering and Discovery Research Institute (KEDRI), Auckland, New Zealand, pp. 101-102 (2003)

12. Nabney, I.: Netlab: Algorithms for Pattern Recognition. Springer, London (2004)

13. Bačić, B.: Using Probability in Estimating the Size of a Test Data Sample. In: 6th International Conference on Hybrid Intelligent Systems (HIS 2006) and the 4th International Conference on Neuro Computing and Evolving Intelligence (NCEI 2006), Auckland, New Zealand, vol. 1, pp. 55-56 (2006)

14. Bacic, B.: A Novel Generic Cluster Based iB-fold Cross-validation, p. 6. Auckland University of Technology, Auckland (2005)

15. Narayanan, A.: Intelligent Bioinformatics and Cancer Systems Biology: The Computational Search for Killer Genes, p. 42. Auckland University of Technology, Auckland (2007)

16. Bacic, B.: Personalised Coaching System, p. 4. AUT, Auckland (2005) 